Pacific Journal of Mathematics

OPEN MAPPINGS ON 2-MANIFOLDS 


\title{
OPEN MAPPINGS ON 2-MANIFOLDS
}

\author{
William D. NathaN
}

This paper will be concerned with the local structure of open, continuous functions $f: M^{2} \rightarrow N^{1}$ from a 2-manifold into the real line or the circle. The two main results are:

Theorem 1. Let $f: M^{2} \rightarrow N^{1}$ be an open mapping, and suppose that $f$ has isolated branch points. Then for each point $p$ in $M^{2}$ there are a neighborhood $U$ of $p$ and a positive integer $d$ (depending on $p$ ) such that $\left.f\right|_{U}$ is topologically equivalent to the real analytic mapping $z \rightarrow \operatorname{Re}\left(z^{d}\right)$.

Theorem 2. If $f: M^{2} \rightarrow N^{1}$ is an open, real analytic mapping, then $f$ has isolated branch points, and (hence) the conclusion of Theorem 1 holds.

2. Definitions and notation. Let $f: X \rightarrow Y$ be any continuous function. Then $f$ is an open mapping if $f(U)$ is an open subset of $Y$ whenever $U$ is an open subset of $X$. If $f^{\prime}: X^{\prime} \rightarrow Y^{\prime}$ is another mapping then $f$ and $f^{\prime}$ are topologically equivalent if there exist homeomorphisms $\alpha: X \rightarrow X^{\prime}, \beta: Y \rightarrow Y^{\prime}$ such that $f^{\prime} \alpha=\beta f$.

If $A$ is any subset of $X$, then $\left.f\right|_{A}$ will denote the restriction of $f$ to $A$.

Let $f: M^{n} \rightarrow N^{p}$ be a mapping from an $n$-manifold into a $p$-manifold, $n \geqq p$. The branch set, $B_{f}$, of $f$ is the subset of $M^{n}$ defined as follows: a point $x$ is in $M^{n}$ - $B_{f}$ if there exists a neighborhood $U$ of $x$ in $M^{n}$ such that $\left.f\right|_{U}$ is topologically equivalent to the canonical projection of $n$-space onto $p$-space.

If $M^{n}$ and $N^{p}$ are differentiable manifolds, and $f: M^{n} \rightarrow N^{p}$ is differentiable, then $R_{q}(f)$ will denote the set of points in $M^{n}$ at which the rank of the Jacobian of $f$ is at most $q, q=0,1, \cdots$. by $\bar{A}$.

The boundary of a set $A$ will be denoted by $\partial A$ and its closure

\section{Proof of Theorem 1. We first need some lemmas.}

LEMMA 3.1. Let $f:[0,1] \times[0,1] \rightarrow[0,1]$ with $\{i\} \times[0,1]=f^{-1}(i)$, $i=0,1$. Suppose there exists a homeomorphism $\alpha:[0,1] \times[0,1] \rightarrow$ $[0,1] \times[0,1]$ which fixes the four vertices of $[0,1] \times[0,1]$ such that $\Pi \alpha=f$, where $\Pi$ is the projection $(x, y) \rightarrow x$. Let $h:\{0\} \times[0,1] \rightarrow$ $\{0\} \times[0,1]$ be any homeomorphism with $h(0,0)=(0,0)$. Then there exists a homeomorphism $\beta:[0,1] \times[0,1] \rightarrow[0,1] \times[0,1]$ such that $\Pi \beta=f$ and $\left.\beta\right|_{\{0\} \times[0,1]}=h$.

Proof. The desired homeomorphism is given by $\beta(x, y)=(f(x, y)$, 
$\left.h^{2} \alpha^{-1}\left(0, \alpha^{2}(x, y)\right)\right)$, where $h^{2}$ and $\alpha^{2}$ are the second coordinate functions of $h$ and $\alpha$, respectively.

Lemma 3.2. Let $f:[0,1] \times[0,1] \rightarrow[0,1]$ be an open mapping. Suppose that the following conditions are satisfied:

(i) $f^{-1}(0)=\{0\} \times[0,1], f^{-1}(1)=\{1\} \times[0,1]$,

(ii) if $g$ is the restriction of $f$ to $(0,1) \times(0,1)$, then $B_{g}=\varnothing$,

(iii) for each point $y$ in the open interval $(0,1), y \neq 1 / 2$ there is a neighborhood of the point $(1, y)$ in $[0,1] \times[0,1]$ on which $f$ is topologically equivalent to the projection of $(0,1] \times(0,1)$ onto $(0,1]$, and for each point $y$ in the open interval $(0,1)$ there is a neighborhood of $(0, y)$ in $[0,1] \times[0,1]$ on which $f$ is topologically equivalent to the projection of $[0,1) \times(0,1)$ onto $[0,1)$,

(iv) for each point $x$ in the open interval $(0,1)$, there is a neighborhood of the point $(x, 0)$ in $[0,1] \times[0,1]$ and a neighborhood of the point $(x, 1)$ in $[0,1] \times[0,1]$ on which $f$ is topologically equivalent to the projection of $(0,1) \times[0,1)$ onto $(0,1)$, and

(v) if $x=0$ or 1 , and $y=0$ or 1 , then there exists a neighborhood of the point $(x, y)$ in $[0,1] \times[0,1]$ on which $f$ is topologically equivalent to the first coordinate projection of $[0,1) \times[0,1)$ onto $[0,1)$.

Then $f$ is topologically equivalent to the first coordinate projection of $[0,1] \times[0,1]$ onto $[0,1]$.

Proof. Let $\Pi:[0,1] \times[0,1] \rightarrow[0,1]$ denote the projection $(x, y) \rightarrow$ $x$. Let $a_{0}=[0,1] \times\{1\}$, and let $b_{0}=[0,1] \times\{0\}$. Let $\left\{y_{n}^{+}\right\}$be a monotone decreasing sequence in the open interval $(1 / 2,1)$ converging to $1 / 2$, and let $\left\{y_{n}^{-}\right\}$be a monotone increasing sequence in the open interval $(0,1 / 2)$ converging to $1 / 2$.

In [7, p. 16 (3.1)], Timourian showed that a proper mapping with empty branch set between manifolds of codimension one is a locally trivial fiber map; hence the restriction of $f$ to $[0,1) \times[0,1]$ is topologically equivalent to the projection of $[0,1) \times[0,1]$ onto $[0,1)$. For each positive integer $n$, let $a_{n}$ be an arc in $[0,1] \times[0,1]$ whose endpoints are $\left(0, y_{n}^{+}\right)$and $\left(1, y_{n}^{+}\right)$, and let $b_{n}$ be an arc in $[0,1] \times$ $[0,1]$ with endpoints $\left(0, y_{n}^{-}\right)$and $\left(1, y_{n}^{-}\right)$satisfying the following conditions (these arcs exist by the above reference and condition (iii)):

(i) the collection $\left\{a_{1}, b_{1}, a_{2}, b_{2}, \cdots\right\}$ is pairwise disjoint, and

(ii) each $a_{i}$ and each $b_{i}$ is mapped by $f$ homeomorphically onto $[0,1]$.

For each $n$, let $A_{n}^{\prime}$ denote the closed 2-cell bounded by $a_{n} \cup$ $a_{n-1} \cup\left(\{0,1\} \times\left[y_{n}^{+}, y_{n-1}^{+}\right]\right)$, and let $B_{n}^{\prime}$ be the closed 2-cell bounded by $b_{n} \cup b_{n-1} \cup\left(\{0,1\} \times\left[y_{n-1}^{-}, y_{n}^{-}\right]\right)$, where $y_{0}^{+}=1$, and $y_{0}^{-}=0$.

Let $\left\{x_{n}\right\}$ be a monotone increasing sequence in the open interval $(0,1)$ converging to 1 , and let $A_{n}=A_{n}^{\prime} \cap f^{-1}\left(\left[x_{n-1}, 1\right]\right)$ and $B_{n}=B_{n}^{\prime} \cap$ 
$f^{-1}\left(\left[x_{n-1}, 1\right]\right)$, where $x_{0}=0$. Finally, let $C_{n}$ denote the closure of

$$
f^{-1}\left(\left[x_{n-1}, x_{n}\right]\right)-\left(\bigcup_{i=1}^{n}\left(A_{i} \cup B_{i}\right)\right) \text {. }
$$

(See figure.)

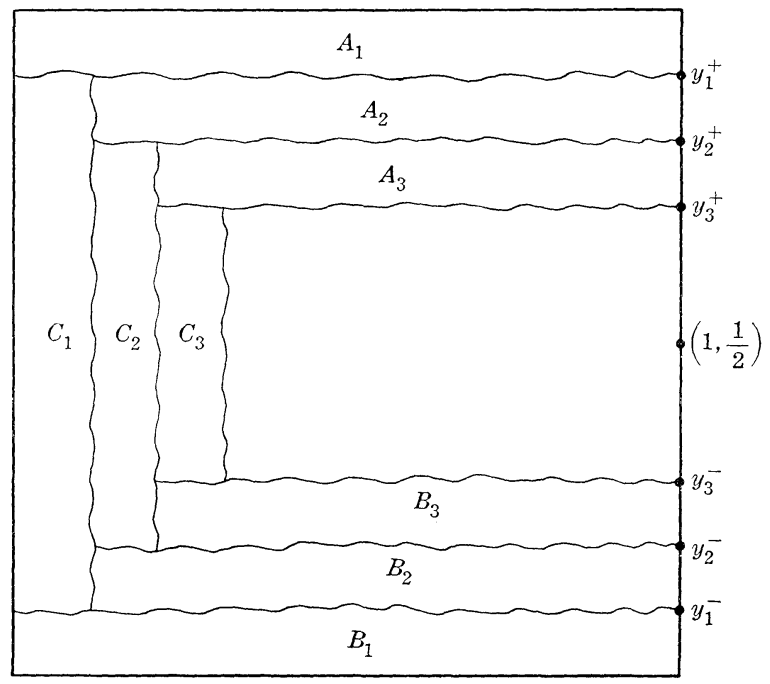

FIGURE 1. phisms

By the aforementioned reference to [7], there exist homeomor-

$$
\begin{aligned}
& h_{n}^{A}: A_{n} \rightarrow\left[x_{n-1}, 1\right] \times\left[y_{n}^{+}, y_{n-1}^{+}\right], \\
& h_{n}^{B}: B_{n} \rightarrow\left[x_{n-1}, 1\right] \times\left[y_{n-1}^{--1}, y_{n}^{-}\right], \text {and } \\
& h_{n}^{C}: C_{n} \rightarrow\left[x_{n-1}, x_{n}\right] \times\left[y_{n}^{-}, y_{n}^{+}\right]
\end{aligned}
$$

such that $\Pi h_{n}^{A}=\left.f\right|_{A_{n}}, \Pi h_{n}^{B}=\left.f\right|_{B_{n}}$ and $\Pi h_{n}^{C}=\left.f\right|_{C_{n}}$. We assume here that $h_{n}^{A}\left(\left(1, y_{i}^{+}\right)\right)=\left(1, y_{i}^{+}\right)$and $h_{n}^{B}\left(\left(1, y_{i}^{-}\right)\right)=\left(1, y_{i}^{-}\right)$for $i=n-1, n$.

Clearly, $h_{n}^{A}$ agrees with $h_{n}^{C}$ on $A_{n} \cap C_{n}$, and $h_{n}^{B}$ agrees with $h_{n}^{C}$ on $B_{n} \cap C_{n}$, so there exists a homeomorphism

$$
\begin{aligned}
h_{n}: A_{n} \cup B_{n} \cup C_{n} & \rightarrow\left(\left[x_{n-1}, 1\right] \times\left[y_{n}^{+}, y_{n-1}^{+}\right]\right) \cup\left(\left[x_{n-1}, 1\right] \times\left[y_{n-1}^{-}, y_{n}^{-}\right]\right) \\
& \cup\left(\left[x_{n-1}, x_{n}\right] \times\left[y_{n}^{-}, y_{n}^{+}\right]\right)
\end{aligned}
$$

such that $\Pi h_{n}=\left.f\right|_{A_{n} \cup B_{n} \cup C_{n}}, n=1,2, \cdots$. Clearly, $h_{n+1}$ agrees with $h_{n}$ on $A_{n+1} \cap A_{n}$ and on $B_{n+1} \cap B_{n}$; moreover, by 3.1 , we may assume that $h_{n+1}$ agrees with $h_{n}$ on $C_{n} \cap\left(A_{n+1} \cup B_{n+1} \cup C_{n+1}\right)$.

Thus, we get a continuous bijection $h ;[0,1] \times[0,1] \rightarrow[0,1] \times$ $[0,1]$ by

$$
h(p)= \begin{cases}h_{n}(p), & \text { if } p \in A_{n} \cup B_{n} \cup C_{n} \\ \left(1, \frac{1}{2}\right), & \text { if } p=\left(1, \frac{1}{2}\right),\end{cases}
$$

satisfying the condition $\Pi h=f$. 
Lemma 3.3. Let $f: M^{n} \rightarrow N^{1}$ be open, let $U \subset M^{n}$ be open, and suppose that $f(U)$ is contained in a closed arc on $N^{1}$. Let $q \in U$, and let $Q$ be a closed subset of $f^{-1}(f(q)) \cap U$. Then no component of $U-Q$ is separated from $\partial U$. (Two sets are separated if their closures are disjoint.)

Proof. If $V$ is a component of $U-Q$, then $V$ is an open connected subset of $M^{n}$, so $f(V)$ is an open interval (whose closure is a closed interval). If $V$ is separated from $\partial U$, then $\partial V \subset Q$. Thus $f(\bar{V})=$ $f(V) \cup f(\partial V)=\{f(q)\} \cup f(V)$ is not compact.

Proof of Theorem 1. Let $U^{\prime}$ be an open disk about $p$ whose closure is a closed disk such that $B_{f} \cap \bar{U}^{\prime} \subset\{p\}$ and $f\left(\bar{U}^{\prime}\right)$ is a closed interval on $N^{1}$. By 3.3, for each point $x$ in $U^{\prime}, f^{-1}(f(x)) \cap U^{\prime}$ contains no simple closed curve. Thus, the closure of each component of $\left(f^{-1}(f(p)) \cap \bar{U}^{\prime}\right)-\{p\}$ is a closed arc with a point of $\partial U^{\prime}$ as one endpoint and either $p$ or a point of $\partial U^{\prime}$ as its other endpoint, and for each point $q$ in $\bar{U}^{\prime}-f^{-1}(f(p))$, each component of $f^{-1}(f(q)) \cap \bar{U}^{\prime}$ is a (possibly degenerate) closed arc each of whose endpoints is on $\partial U^{\prime}$.

Let $A_{1}, A_{2}, \cdots, A_{k}$ be the closures of the component ares of $\left(f^{-1}(f(p)) \cap \bar{U}^{\prime}\right)-\{p\}$ which have $p$ as an endpoint, and let $\alpha_{i}$ be the endpoint of $A_{i}$ on $\partial U^{\prime}$. (There are clearly finitely many such $A_{i}$.) Orient $\partial U^{\prime}$, and index the $a_{i}$ in order of increasing argument.

Let $R_{1}^{\prime}, \cdots, R_{k}^{\prime}$ be the components of $U^{\prime}-\bigcup_{i=1}^{k} A_{i}$, indexed so that $a_{j} \in \bar{R}_{i}^{\prime}$ if, and only if, $j=i$ or $j \equiv(i+1)(\bmod k)$.

For each $i=1,2, \cdots, k$, there exist a neighborhood $U_{i}$ of $a_{i}$ and a homeomorphism $\alpha_{i}^{\prime}: U_{i} \rightarrow E^{2}$ such that $\Pi \alpha_{i}^{\prime}=\left.f\right|_{U_{i}}$, where $\Pi: E^{2} \rightarrow$ $E^{1}$ is projection onto the first coordinate. We may as well assume that $U_{i} \cap U_{j}=\varnothing$ when $i \neq j, \alpha_{i}^{\prime}\left(U_{i} \cap \partial U^{\prime}\right)$ is the $x$-axis, and $\Pi \alpha_{i}^{\prime}(q)>$ 0 for each $q \in R_{i}^{\prime} \cap U_{i}$.

Let $\left\{Y_{\alpha}\right\}$ be the collection of all components of $\left(f^{-1}(f(p)) \cap \bar{U}^{\prime}\right)-$ $\{p\}$ which do not have $p$ as a limit point, and let $Y=\cup Y_{\alpha}$. Clearly, $p$ is not a limit point of $Y$, so there exists a neighborhood $U^{\prime \prime}$ of $p$ such that

$$
\left(\bigcup_{i=1}^{k} U_{i}\right) \cap \bar{U}^{\prime}=\left(\bigcup_{i=1}^{k} U_{i}\right) \cap \bar{U}^{\prime \prime} \quad \text { and } \quad \bar{U}^{\prime \prime} \cap f^{-1}(f(p))=\bigcup_{i=1}^{k} A_{i} .
$$

Let $R_{i}=R_{i}^{\prime} \cap U^{\prime \prime}$, and fix $i$. We claim that there exists a point $t_{i} \in\left(\bar{R}_{i} \cap \partial U^{\prime \prime} \cap U_{i}\right)-\left\{a_{i}\right\}$ such that if $x$ is any point in $\left(\bar{R}_{i} \cap \partial U^{\prime \prime} \cap U_{i}\right)$ with $0<\Pi \alpha_{i}^{\prime}(x) \leqq \Pi \alpha_{i}^{\prime}\left(t_{i}\right)$ and $X$ is the component arc of $f^{-1}(f(x)) \cap \bar{U}^{\prime \prime}$ containing $x$, then $x^{\prime} \in U_{j}$, where $j \equiv(i+1)(\bmod k)$, and $x^{\prime}$ is the other endpoint of $X$. For suppose not. Then there exists a sequence $\left\{x_{n}\right\}$ in $\left(\bar{R}_{i} \cap \partial U^{\prime \prime} \cap U_{i}\right)-\left\{a_{i}\right\}$ converging to $a_{i}$ such that $x_{n}^{\prime} \notin U_{j}$, for each $n$. There is a convergent subsequence of $\left\{x_{n}^{\prime}\right\}$ whose limit is in 
$f^{-1}(f(p)) \cap \partial U^{\prime \prime}$ but is not in $\bigcup_{i=1}^{k} A_{i}$. This contradicts $(*)$, above.

Write $f\left(\bar{U}^{\prime \prime}\right)$ as the closed interval $\left[a^{\prime}, b^{\prime}\right]$, where $a^{\prime}<f(p)<b^{\prime}$. We may assume that $f(p)<f\left(t_{i}\right)<b^{\prime}$ if $i$ is even, and $a^{\prime}<f\left(t_{i}\right)<$ $f(p)$ if $i$ is odd. Note that $k$ is even. Let $b=\min \left\{f\left(t_{2}\right), f\left(t_{4}\right), \cdots, f\left(t_{k}\right)\right\}$, and let $a=\max \left\{f\left(t_{1}\right), f\left(t_{3}\right), \cdots, f\left(t_{k-1}\right)\right\}$. Finally, let $U=\bar{U}^{\prime \prime} \cap f^{-1}([a, b])$, let $d=k / 2$, and assume $a=0$ and $b=1$.

If $S_{i}=\bar{R}_{i} \cap U$, then $\left.f\right|_{S_{i}}$ satisfies the hypotheses of 3.2 . Note that $f\left(S_{i}\right)=[f(p), b]$ if $i$ is even, and $f\left(S_{i}\right)=[a, f(p)]$ if $i$ is odd, so by 3.2 there exists a homeomorphism $\alpha_{i}: S_{i} \rightarrow[-1,1] \times[0,1]$ such that $\left.f\right|_{S_{i}}=\Pi^{\prime} \alpha_{i}$, where $\Pi^{1}:[-1,1] \times[0,1] \rightarrow[0,1]$ is the natural projection. We may assume that $\alpha_{i}(p)=(0,1)$, and that if $j \equiv(i+1)(\bmod k)$, then $\alpha_{i}\left(A_{j}\right)=\alpha_{j}\left(A_{j}, i=1,2, \cdots, k\right.$.

Let $X_{1}, X_{2}, \cdots, X_{k}$ be disjoint copies of $[-1,1] \times[0,1]$ indexed so that $\alpha_{i}\left(S_{i}\right)=X_{i}$. Form the disjoint union $X=X_{1}+X_{2}+\cdots+X_{k}$. Now, make the following identifications on $X$ : if $x_{j} \in \alpha_{j}\left(A_{j}\right)$ and $j \equiv$ $(i+1)(\bmod k)$, then identify $x_{j}$ with $\alpha_{i} \alpha_{j}^{-1}\left(x_{j}\right)$.

Let $X / \alpha$ denote the resulting identification space, and let $P_{\alpha}: X \rightarrow$ $X / \alpha$ be the quotient map. Let $\alpha: U \rightarrow X / \alpha$ by $\alpha(u)=P_{\alpha} \alpha_{i}(u)$, if $u \in$ $S_{i}$. Then $\alpha$ is a homeomorphism.

Let $\Pi_{i}: X_{i} \rightarrow[0,1]$ denote the projection mapping, $i=1,2, \cdots, k$, and let $\sum_{i=1}^{k} \Pi_{i}: X \rightarrow[0,1]$ by $\left(\sum_{i=1}^{k} \Pi_{i}\right)\left(x_{j}\right)=\Pi_{j}\left(x_{j}\right)$, where $x_{j} \in X_{j}$. Then $\sum_{i=1}^{k} \Pi_{i}$ can be factored through $P_{\alpha}$, obtaining a map $G: X / \alpha \rightarrow$ $[0,1]$ such that $G P_{\alpha}=\sum_{i=1}^{k} \Pi_{i}$. But then $\left.f\right|_{U}=G \alpha$; i.e., $\left.f\right|_{U}$ is topologically equivalent to $G$. Theorem 1 follows.

In [8, Theorem 1], Tôki gives a topological characterization of mappings on a 2-manifold which are pseudo-harmonic. His results imply that if $f: M^{2} \rightarrow N^{1}$ is an open map with $B_{f}$ discrete, then $f$ is topologically equivalent to a harmonic function. It is well known (see, for example, [5, pp. 8-9]) that harmonic functions have the local structure of (our) Theorem 1. The advantage of the present approach must lie in its directness and brevity.

4. The real analytic case. In this section we prove Theorem 2 . A few facts from the theory of analytic varieties will be needed, and we include them here for completeness. For the reader who is unfamiliar with the terminology, we recommend [6].

LEMMA 4.1. Every real analytic variety is locally connected.

LEMMA 4.2. If $V$ is a k-dimensional variety in the real analytic manifold $M$, then for every point $p \in V$, there is a neighborhood $U$ of $p$ in $M$ such that $V \cap U$ is the disjoint union $V^{\prime}+V^{\prime \prime}$, where $V^{\prime}$ is a $k$-dimensional real analytic submanifold of $U$, and $V^{\prime \prime}$ is a variety in $U$ of dimension at most $k-1$. 
The proof of 4.2 is in [1].

If $f: M \rightarrow N$ is a real analytic mapping, then clearly the sets $f^{-1}(y), y \in N$, are varieties, as are the sets $R_{q}(f)$, defined in $\S 2$. Moreover, it was proved by Church [2, p. $88(1.3)]$ that $\operatorname{dim}\left(f\left(R_{q}(f)\right)\right) \leqq$ $q, q=0,1, \cdots$.

LEMMA 4.3. Let $f: M^{n} \rightarrow N^{1}$ be a proper real analytic mapping. Then $f\left(R_{0}(f)\right)$ is discrete.

Proof. Let $y$ be a limit point of $f\left(R_{0}(f)\right)$. Let $\left\{y_{n}\right\}$ be a sequence in $f\left(R_{0}(f)\right)$, converging to $y$, and let $x_{n}$ be a point in $f^{-1}\left(y_{n}\right) \cap R_{0}(f)$, $n=1,2, \cdots$. By the properness of $f$, the sequence $\left\{x_{n}\right\}$ has an accumulation point; call it $x$. Since $R_{0}(f)$ is closed, $x \in R_{0}(f)$. Then, if $U$ is any neighborhood of $\left.x, U \cap R_{0}(f)-\{x\}\right) \neq \varnothing$. Then, by the 0-dimensionality of $f\left(R_{0}(f)\right.$ ) (see remark, above), $U \cap R_{0}(f)$ is not connected; i.e., $R_{0}(f)$ is not locally connected at $x$, contradicting 4.1.

Proof of Theorem 2. Let $D$ be an open disk in $M^{2}$ with compact closure, and let $g=\left.f\right|_{D}$. Then $g\left(R_{0}(g)\right)$ is discrete by 4.3. Let $q \in$ $g\left(R_{0}(g)\right)$. It suffices to show that $g^{-1}(q) \cap B_{g}$ is discrete.

Since $\operatorname{dim}\left(g^{-1}(q)\right)=1$, we can remove from $g^{-1}(q)$, by 4.2 , a discrete set $A^{\prime}$ such that $g^{-1}(q)-A^{\prime}$ is a 1-dimensional real analytic submanifold of $D$. Let $p$ be a point in $g^{-1}(q)-A^{\prime}$, and let $U \subset D$ be a coodinate chart about $p$ with real analytic coordinates $(x, y)$ such that $g^{-1}(q)$ is the $y$-axis and $g\left(R_{0}(g) \cap U\right)=\{q\}$. Let $h=\left.f\right|_{U}$. By Fox's Spoke Theorem $\left[4\right.$, p. 347 (5.1)], it suffices to show that $h^{-1}(q) \cap B_{h}$ is discrete.

Suppose $W$ is an open disk about a point on $h^{-1}(q) \cap U$ such that each horizontal line in $W$ given by $y=$ constant is mapped homeomorphically by $h$ onto $h(W)$. Then $W \cap B_{h}=\varnothing$. For define $F: W \rightarrow$ $h(W) \times R$ by $F(x, y)=(h(x, y), y)$; this will give the desired equivalence.

For such fixed $\bar{y}$, consider the set $X(\bar{y})$ of points $(x, \bar{y})$ at which $\left.h\right|_{y=\bar{y}}$ is not interior; i.e., the set of points at which $\left(\partial / \partial_{X}\right)\left(\left.h\right|_{y=\bar{y}}\right)$ changes sign. Now, $X=\bigcup_{\bar{y}} X(\bar{y})$ is contained in the set $A=\{(x, y)$ : $(\partial h / \partial x)(x, y)=0\} . \quad A$ is clearly a variety, and the openness of $f$ implies that $\operatorname{dim}(A) \leqq 1$. If $A$ is discrete, then the comment in the last paragraph applies and there is nothing to prove. Hence, we may as well assume that $\operatorname{dim}(A)=1$. Let $E$ be the set of points at which $A$ is not a 1 -submanifold.

Consider the intersection of $A$ with the $y$-axis. It is a variety of dimension at most one. If it has dimension zero (i.e., if it is discrete), then there is nothing to prove. So suppose it is 1-dimensional, 
in which case it is the entire $y$-axis. Now, the openness of $h$ implies that all points of $U$ with positive abscissa map on one side of $q$, and the points with negative abscissa map on the other side of $q$. Hence, $\partial h / \partial x$ does not change sign at any point of the $y$-axis. Thus, every point on the $y$-axis which is not in $E$ has a neighborhood disjoint from $X$, and $E$ is discrete.

For more extensive results on the topological properties of real analytic mappings, see [3].

\section{REFERENCES}

1. F. Bruhat and H. Whitney, Quelques propriétés fondamentales des ensembles analytiques-réels, Comment. Math. Helv. 33 (1959), 132-160.

2. P. T. Church, Differentiable open maps on manifolds, Trans. Amer. Math. Soc., 109 (1963), 87-100.

3. P. T. Church and W. D. Nathan, Real analytic maps on manifolds, J. Math. Mech., 18 (1969), 19-35.

4. W. C. Fox, The critical points of Peano-interior functions defined on 2-manifolds, Trans. Amer. Math. Soc., 83 (1956), 338-370.

5. M. Morse, Topological Methods in the Theory of Functions of a Complex Variable, Princeton University Press, Princeton, 1947.

6. R. Narasimhan, Introduction to the Theory of Analytic Spaces, Springer Verlag Lecture Notes in Mathematics, No. 25, Berlin, 1966.

7. J. Timourian, Singular Fiberings of Manifolds, Doctoral Dissertation, Syracuse University, 1967.

8. Y. Tôki, A topological characterization of pseudoharmonic functions, Osaka Math. J., 3 (1951), 101-122.

Received April 8, 1970 and in revised form September 24, 1971. This work was part of the author's doctoral thesis, completed in 1968 at Syracuse University. 



\section{PACIFIC JOURNAL OF MATHEMATICS}

\section{EDITORS}

\author{
H. SAMELSON \\ Stanford University \\ Stanford, California 94305

\section{R. HoвBY} \\ University of Washington \\ Seattle, Washington 98105
}

\section{J. DugundJI}

Department of Mathematics University of Southern California Los Angeles, California 90007

\author{
RichaRd ARENS \\ University of California \\ Los Angeles, California 90024
}

\section{ASSOCIATE EDITORS}
E. F. BECKENBACH
B. H. NEUMANN
F. WOLF
K. YoshidA

\section{SUPPORTING INSTITUTIONS}

\author{
UNIVERSITY OF BRITISH COLUMBIA \\ CALIFORNIA INSTITUTE OF TECHNOLOGY \\ UNIVERSITY OF CALIFORNIA \\ MONTANA STATE UNIVERSITY \\ UNIVERSITY OF NEVADA \\ NEW MEXICO STATE UNIVERSITY \\ OREGON STATE UNIVERSITY \\ UNIVERSITY OF OREGON \\ OSAKA UNIVERSITY
}

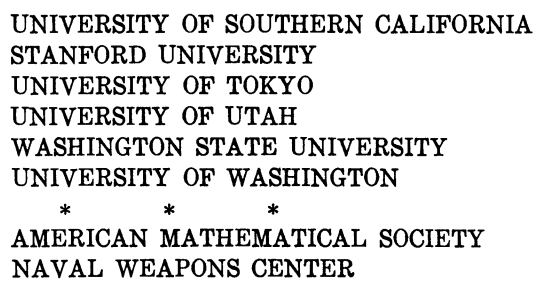

The Supporting Institutions listed above contribute to the cost of publication of this Journal, but they are not owners or publishers and have no responsibility for its content or policies.

Mathematical papers intended for publication in the Pacific Journal of Mathematics should be in typed form or offset-reproduced, (not dittoed), double spaced with large margins. Underline Greek letters in red, German in green, and script in blue. The first paragraph or two must be capable of being used separately as a synopsis of the entire paper. The editorial "we" must not be used in the synopsis, and items of the bibliography should not be cited there unless absolutely necessary, in which case they must be identified by author and Journal, rather than by item number. Manuscripts, in dup icate if possible, may be sent to any one of the four editors. Please classify according to the scheme of Math. Rev. Index to Vol. 39. All other communications to the editors should be addressed to the managing editor, Richard Arens, University of California, Los Angeles, California, 90024.

50 reprints are provided free for each article; additional copies may be obtained at cost in multiples of 50 .

The Pacific Journal of Mathematics is published monthly. Effective with Volume 16 the price per volume (3 numbers) is $\$ 8.00$; single issues, $\$ 3.00$. Special price for current issues to individual faculty members of supporting institutions and to individual members of the American Mathematical Society: $\$ 4.00$ per volume; single issues $\$ 1.50$. Back numbers are available.

Subscriptions, orders for back numbers, and changes of address should be sent to Pacific Journal of Mathematics, 103 Highland Boulevard, Berkeley, California, 94708.

PUBLISHED BY PACIFIC JOURNAL OF MATHEMATICS, A NON-PROFIT CORPORATION

Printed at Kokusai Bunken Insatsusha (International Academic Printing Co., Ltd.), 270, 3-chome Totsuka-cho, Shinjuku-ku, Tokyo 160, Japan. 


\section{Pacific Journal of Mathematics}

\section{Vol. 41, No. 2 December, 1972}

Tom M. (Mike) Apostol, Arithmetical properties of generalized Ramanujan sums .......................................... 281

David Lee Armacost and William Louis Armacost, On p-thetic groups ........ 295

Janet E. Mills, Regular semigroups which are extensions of groups .......... 303

Gregory Frank Bachelis, Homomorphisms of Banach algebras with minimal ideals ................................................ 307

John Allen Beachy, A generalization of injectivity .................. 313

David Geoffrey Cantor, On arithmetic properties of the Taylor series of rational functions. II.........................................

Václáv Chvátal and Frank Harary, Generalized Ramsey theory for graphs. III.

Small off-diagonal numbers .................................. 335

Frank Rimi DeMeyer, Irreducible characters and solvability of finite groups . . . . 347

Robert P. Dickinson, On right zero unions of commutative semigroups........ 355

John Dustin Donald, Non-openness and non-equidimensionality in algebraic

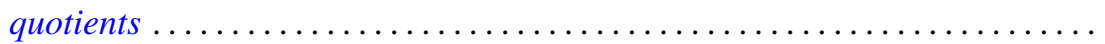

John D. Donaldson and Qazi Ibadur Rahman, Inequalities for polynomials with a prescribed zero ........................................ 375

Robert E. Hall, The translational hull of an $N$-semigroup ................ 379

John P. Holmes, Differentiable power-associative groupoids.............. 391

Steven Kenyon Ingram, Continuous dependence on parameters and boundary data for nonlinear two-point boundary value problems .

Robert Clarke James, Super-reflexive spaces with bases ..........

Gary Douglas Jones, The embedding of homeomorphisms of the plane in

continuous flows...............................

Mary Joel Jordan, Period $H$-semigroups and $t$-semisimple periodic

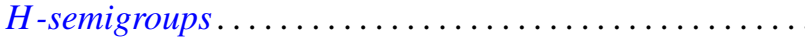

Ronald Allen Knight, Dynamical systems of characteristic 0

Kwangil Koh, On a representation of a strongly harmonic ring by sheaves...

Hui-Hsiung Kuo, Stochastic integrals in abstract Wiener space. ..

Thomas Graham McLaughlin, Supersimple sets and the problem of extending a

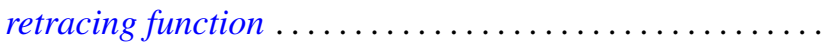

William Nathan, Open mappings on 2-manifolds .

M. J. O'Malley, Isomorphic power series rings

Sean B. O'Reilly, Completely adequate neighborhood systems and metrization

Qazi Ibadur Rahman, On the zeros of a polynomial and its derivative...

Russell Daniel Rupp, Jr., The Weierstrass excess function ..

Hugo Teufel, A note on second order differential inequalities and functional

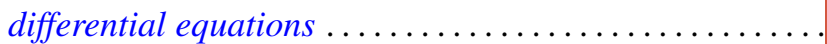

M. J. Wicks, A general solution of binary homogeneous equations over free 